\title{
Non-Transitive Binary Relation of Preference in the Case of Random Value Functions, Derived from REPOMP
}

\author{
Natalia D. Nikolova ${ }^{1}$, Snejana Ivanova $^{1}$, Gergana Georgieva $^{1}$, Kiril Tenekedjiev $^{1}$ \\ ${ }^{1} \mathrm{~N}$. Vaptsarov Naval Academy, Varna, Bulgaria \\ natalianik@gmail.com, sneji_di@abv.bg,gvg_123@abv.bg, Kiril.Tenekedjiev@fulbrightmail.org
}

\begin{abstract}
This work analyses decision making situations, where the quantity of the value function associated with the alternatives is a random number with known distribution. The main contribution of the paper is that alternatives are grouped into pseudo indifference classes, where the alternatives are indifferent to at least one of the other alternatives in the class. However, not all elements in the set are indifferent to each other, unlike classical indifference classes. Since the resulting relation of strict preference over pseudo indifference classes turns out to be non-transitive, it is demonstrated both in theory and in terms of an example that it is strongly dependent on the significance level of comparisons in order to allocate alternatives into groups.
\end{abstract}

\section{Keywords}

indifference classes, nontransitivity, hypothesis testing, ranking

\section{Introduction}

The main task of decision analysis, and more particularly multi-criteria decision making, is to make a choice under uncertainty. Here, each alternative is associated with a consequence. In order to be rational, the decision maker (DM) must obey certain properties of her binary relations of preference, one of which being transitivity (in its different forms). Assume there is a set of $n$ number of objects $B=\left\{b_{1}, b_{2}, b_{3}, \ldots, b_{n}\right\}$, where $b_{i}$ and $b_{j}$ are two objects from that set. Also assume that the relative preference over the alternatives is measured by a value function $v($.$) [Ekenberg, 1998]. Then there exists a$ theorem claiming that if a series of rationality rules apply for the DM's preferences, then the following dependencies hold for the function $v():. v\left(b_{i}\right) \geq v\left(b_{j}\right) \Leftrightarrow b_{i}$ $\succsim b_{j}$ and $v\left(b_{i}\right)>v\left(b_{j}\right) \Leftrightarrow b_{i} \succ b_{j}$ (where $\succsim$ is the binary relation "at least as preferred as", and $\succ$ stands for the binary relation "more preferred than", both defined over objects). The opposite statement is trivially true, namely that if the value function corresponds to the above dependencies, then the binary relations of preference correspond to the axioms of rational choice, since they coincide in terms of properties with the binary relations $\geq$ and $>$ over real numbers.
For many alternatives, the result of the comparison may be brought down to strict preference over groups of indifferent objects, called indifference classes. For some alternatives, however, especially when using computer intensive methods, the function $v($.$) takes random values$ corresponding to a known distribution. Such a setup is present for the method REPOMP (Randomized Expert Panel Opinion Marginalizing Procedure) [Tenekedjiev, Kamenova, Nikolova, 2004]. It offers an approach to analyze the subjective opinion of an expert panel, and a hierarchy of criteria is constructed for the sake of the analysis.

This paper offers a method to order alternatives with random value functions of the above described type. Unfortunately, the resulting binary relation of preference would turn out to be non-transitive and would depend on the significance level of the comparison. In order to compare multiple alternatives, pseudo indifference classes are introduced, where the alternatives are indifferent to at least one of the other alternatives in the class. However, not all elements in the set are indifferent to each other, unlike classical indifference classes.

In what follows, Section 2 presents the properties of binary relations of preference. Section 3 discusses the theory of indifference classes. Section 4 solves the task to order alternatives with random value functions. Section 5 offers a numerical example for the use of the newly proposed approach in solving a task using REPOMP.

\section{Properties of classical binary relations of preference}

Let $B$ be a set of objects $B=$ $=\left\{b_{1}, b_{2}, b_{3}, \ldots\right\}$. Despite the notation, it may be assumed that $B$ is either finite, infinite countable, or infinite uncountable set. For example, the set of students in a school class, the set of teams in the Premier League (the English soccer championship) last year, the set of real number, the set of consequences in a decision tree, etc. Let's also analyze a condition $R$ for any two elements from the set $B$. Let's define a logical function for an ordered pair of two arguments from the set $B$, which takes two values - true and false - at a condition $R$ :

$$
f\left(b_{i}, b_{j}\right)=\left\{\begin{array}{l}
\text { true, if } R \text { holds, } \\
\text { false, if } R \text { does not hold. }
\end{array}\right.
$$


The domain of $f\left(b_{i}, b_{j}\right)$ is

$$
\left\{\left(b_{i}, b_{j}\right) \mid b_{i}, b_{j \in B}\right\} .
$$

This function is called a binary relation, defined over the set $B$. The notation $b_{i} R b_{j}$ stands for the situation where the function $f\left(b_{i}, b_{j}\right)=$ "true", whereas $b_{i} R b_{j}$ stands for the situation, where the function $f\left(b_{i}, b_{j}\right)=$ "false". It is obvious that for any ordered pair of objects $b_{i}$ and $b_{j}$ one of the following two conditions hold:

$$
b_{i} R b_{j} \text { or } b_{i} R b_{j} \text {. }
$$

In other words, (3) says that for any ordered pair of objects from the set $B$ the condition $R$ either holds or not. For example, let's analyze the relation "thicker than" defined over the set of books in a library. Then for any two books $A$ and $B$ from that set either $\mathrm{A}$ is thicker than $B$, or $A$ is not thicker than $B$ (which means that $A$ is either as thick as $B$, or $B$ is ticker). The binary relations may have other properties as well, such as transitivity, symmetry, asymmetry, reflexivity, irreflexivity, comparability, etc.

The result of comparing two objects may be presented by three relations: "more preferred according to the DM than", "equally preferred according to the DM to", and "at least as preferred according to the DM as". These three binary relations are usually called preference orders [Daniels, 1999; Sung, 2000]. Regardless of the name, these relations are not always orders, i.e. they are not always transitive:

A) The relation "is more preferred according to the DM than" shall be shortly called strict preference and shall be denoted by $\succ$. The dependence $b_{i} \succ b_{j}$ means that if the DM is offered a choice between these two objects, then she will be quite dissatisfied if forced to take $b_{j}$ later on;

B) The relation "is equally preferred according to the DM to" shall be shortly called indifference and shall be denoted by $\sim$. The dependence $b_{i} \sim b_{j}$ means that if the DM is offered a choice between the two objects, then she will not be disappointed if either forced to take $b_{i}$ or $b_{j}$;

C) The relation "is at least as preferred according to the DM as" shall be shortly called weak preference and shall be denoted by $\succsim$. The dependence $b_{i} \succsim b_{j}$ means that if the DM is offered the choice between the two objects, then she will not be disappointed if forced to take $b_{j}$.

The necessity to choose the best alternative imposes rationality in the preferences of the DM. What follows are nine requirements to the binary relations $\succ$ and $\sim$, which guarantee that the DM has rational preferences, expressed by her value system:

1) The strict preference of the rational DM must be transitive (support on this seemingly axiomatic rule is provided in [French, 1993; Sugden, 1999]):

if for any three objects $b_{i}, b_{j}, b_{k}$ the DM holds $b_{i} \succ b_{j}$ and $b_{j} \succ b_{k}$, then she must also hold $b_{i} \succ b_{k}$.

2) The strict preference of the rational DM must be asymmetric:

if for any two objects $b_{i}, b_{j}$ the DM holds $b_{i} \succ b_{j}$, then she must also hold $b_{j} \nsucc b_{i}$.

3) The strict preference of the rational DM must be irreflexive:

there is no object $b_{i}$ such that the DM holds $b_{i} \succ$

$$
b_{i} \text {. }
$$

4) The indifference of the rational DM must be transitive:

$$
\begin{aligned}
& \text { if for any objects } b_{i}, b_{j}, b_{k} \text { the DM holds } b_{i} \sim b_{j} \\
& \text { and } b_{j} \sim b_{k}, \\
& \text { then she must also hold } b_{i} \sim b_{k} \text {. }
\end{aligned}
$$

5) Indifference of the rational DM must be symmetric:

if for any two objects $b_{i}, b_{j}$ the DM holds $b_{i} \sim b_{j}$,

$$
\text { then she must also hold } b_{j} \sim b_{i} \text {. }
$$

6) Indifference of the rational DM should be reflexive:

for each object $b_{i}$ the DM should hold $b_{i} \sim b_{i}$.

7) Mutual transitivity of strict preference and indifference:

$$
\begin{gathered}
\text { if for any objects } b_{i}, b_{j}, b_{k} \text { the DM holds } b_{i} \succ b_{j} \\
\text { and } b_{j} \sim b_{k}, \\
\text { then she must hold } b_{i} \succ b_{k} .
\end{gathered}
$$

8) Mutual transitivity between indifference and strict preference:

if for any objects $b_{i}, b_{j}, b_{k}$ the DM holds

$$
\begin{gathered}
b_{i} \sim b_{j} \text { and } b_{j} \succ b_{k}, \\
\text { then it should also hold } b_{i} \succ b_{k} .
\end{gathered}
$$

9) Trichotomy of strict preference and indifference

for any two objects $b_{i}, b_{j}$ the DM should hold exactly one of the following:

$$
b_{i} \succ b_{j}, b_{j} \succ b_{i}, b_{i} \sim b_{j} .
$$

The rationality of preferences, expressed by $\succsim$, is defined in a set of five axioms:

1) Comparability: 
For each two objects $b_{i}, b_{j}$ the DM thinks that exactly one of the following three statements holds:

$b_{i} \succsim b_{j}$ and $b_{j} \succsim b_{i} ; b_{j} \succsim b_{i}$ and $b_{i} \underset{\sim}{\sim} b_{j} ; b_{i} \succsim b_{j}$ and $b_{j} \succsim b_{i}$.

2) Transitivity:

If for any three objects $b_{i}, b_{j}, b_{k}$ the DM thinks that

$$
b_{i} \succsim b_{j} \text { and } b_{j} \succsim b_{k},
$$

then she must also think that

$$
b_{i} \succsim b_{k} \text {. }
$$

\section{3) Reflexivity:}

for all objects $b_{i}$ the DM should think that $b_{i} \succsim$ $b_{i}$

4) Consistency of $\sim$ and $\succsim$

or any two objects $b_{i}, b_{j}$ the DM thinks that $b_{i} \sim b_{j}$ if and only if she holds $b_{i} \succsim b_{j}$ and $b_{j} \succsim b_{i}$.

5) Consistency of $\succ$ and $\succsim$

for any two objects $b_{i}, b_{j}$ the DM thinks that $b_{i} \succ$ $b_{j}$

if and only if she holds $b_{j} \succsim b_{i}$ and $b_{i} \succsim b_{j}$.

\section{Indifference classes}

Let $I_{1}, I_{2}, \ldots, I_{m}$ be a partition of the set $B$ of objects:

$$
\begin{aligned}
& B \equiv I_{1} \cup I_{2} \cup \ldots \cup I_{m}, \\
& I_{i} \cap I_{j} \equiv \varnothing, \forall i \neq j .
\end{aligned}
$$

All elements that belong to a given subset $I_{i}, i=1,2, \ldots$, $m$, must be equally preferred by the DM. Such a subset is called indifference class. Let $b_{i}$ be available from the indifference class $I_{j}$. Then it is possible to identify the elements of the subset $I_{j}$ and be called the indifference class of $b_{i}$. An indifference class $I\left(b_{i}\right)$ may be identified for each object $b_{i}$ from the set $B$, as all objects from $B$ that are indifferent to $b_{i}$ :

$$
I_{j} \equiv I\left(b_{i}\right) \equiv\left\{b_{k} \in B \mid b_{k} \sim b_{i}\right\} .
$$

The binary relation $\succ_{i}$ - "the indifference class $I_{k}$ is preferred over the indifference class $I_{j}$ " may be defined over the set of indifference classes $\left\{I_{1}, I_{2}, \ldots, I_{m}\right\}$. This means that according to the DM, all elements in $I_{k}$ are strictly preferred over all elements of $I_{j}$ :

$$
\begin{aligned}
I_{k} \succ_{i} I_{j} \Leftrightarrow b_{p} \succ b_{q}, & \\
& \forall b_{p} \in I_{k}, \forall b_{q} \in I_{j} .
\end{aligned}
$$

The essence of the indifference classes approach is to find the preference order of those objects, as follows:

$$
I_{1} \succ_{i} I_{2} \succ_{i} \quad \ldots \succ_{i} I_{m}
$$

It is possible to prove that if the preferences of the DM obey the axioms of rational choice (see Section 2), then the ranking of objects using the indifference classes would be rational. The following statements hold:

- if two indifference classes have a common object, then those coincide, i.e.

$$
\text { if } I\left(b_{i}\right) \cap I\left(b_{j}\right) \equiv \varnothing \text {, then } I\left(b_{i}\right) \equiv I\left(b_{j}\right)
$$

- if two objects are mutually indifferent, then their indifference classes coincide, i.e.

$$
\text { if } b_{i} \sim b_{j} \text {, then } I\left(b_{i}\right) \equiv I\left(b_{j}\right) \text {; }
$$

- if an object is strictly preferred over another, then each member of the indifference class of the first object is also strictly preferred over each member of the indifference class of the second object, i.e.

$$
\begin{aligned}
& \text { if } b_{i} \succ b_{j}, \text { then } b_{p} \succ b_{q}, \\
\forall & b_{p} \in I\left(b_{i}\right), \forall b_{j} \in I\left(b_{j}\right) .
\end{aligned}
$$

The indifference classes method [Gilboa, Schmeidler, Wakker, 2001; Ghirardato, Marinacci, 2000] is the best nonfunctional method to express the preferences of the DM over a given set of objects $B$. It is a compact and clear approach, constructed on the grounds of the relations "strict preference" and "indifference", which are highly intuitive. There is a mathematical elegancy in the proofs that use the aparatus of indifference classes, which is why they are often used in specialized research works in decision analysis. The only drawback of the approach comes from the fact that the DM wishes to express preferences over objects instead of sets of objects.

\section{Ordering of alternatives in the case of random value functions}

Assume there are $c$ number of alternatives $b_{1}, b_{2}, \ldots, b_{c}$. Let the value function $v_{k}=v\left(b_{k}\right)$ of the $k$-th alternative $b_{k}$ be a random variable, with $\mathrm{PDF}-f_{k}(v)$. Let $\operatorname{med}_{k}$ be the median of the random variable $b_{k}$. That is,

$$
0.5=\int_{-\infty}^{\operatorname{med}_{k}} f_{k}(v) d v
$$

Let $\operatorname{med}_{s}<$ med $_{l}$. Let's construct a statistical test regarding the equality of $v_{s}$ and $v_{l}$, whereas the alternative 
hъpothesis is $v_{s}<v_{k}$. Let the hypothesis $H_{0}$ is tested at a given significance level $\alpha$. If med $_{s}=$ med $_{l}$, then $p_{\text {value }}$ may be set to 0.5 . Otherwise, $p_{\text {value }}$ between the alternatives $s$ and $l$ may be calculated using the formula

$$
p_{\text {value }}(s, l)=\int_{-\infty}^{+\infty} \int_{-\infty}^{v_{s}} f_{l}\left(v_{l}\right) d v_{l} d v_{s}
$$

Often, $f_{k}(v)$ is approximated as a sum of delta functions with an area of $1 / n_{k}$, centered over experimentally derived realizations of the random variable $v_{k}: v_{k, 1}, v_{k, 2}, \ldots, v_{k, n_{k}}$. Then, $p_{\text {value }}$ may be simplified as:

$$
\begin{aligned}
& p_{\text {value }}(s, l)=\frac{1}{n_{s} n_{l}} \sum_{j=1}^{n_{s}} \sum_{\substack{i=1 \\
v_{s, j}>v_{l, i}}}^{n_{l}} 1= \\
& =\frac{1}{n_{s} n_{l}} \sum_{j=1}^{n_{s}} \sum_{i=1}^{n_{l}} L\left(v_{s, j}>v_{l, i}\right)
\end{aligned}
$$

where $L$ (condition) stands for 1 if the condition in the brackets is true, and 0 if the condition in the brackets is not true. The value of $p_{\text {value }}$ is in fact the number of pairs from the realization of the two random variables $v_{s}$ and $v_{k}$, where the "small" variable is larger than the "large" variable, divided by the number of all possible comparisons. As it is evident from the above formula, this is a non-parametric test that is based on almost no assumptions.

If $p_{\text {value }}(s, 1)<\alpha$, then the null hypothesis is rejected, thus the random variable $v_{k}$ is less than the random variable $v_{l}$. This implies that $b_{l} \succ b_{s}$. By analogy, if $p_{\text {value }}(s, 1) \geq \alpha$, then the null hypothesis fails to be rejected, thus the random variable $v_{k}$ is considered equal to $v_{l}$. This implies that $b_{l} \sim b_{s}$. Evidently, the so-defined indifference and weak preference would not be transitive, and the mutual transitivities also do not hold, i.e. formulae (7), (10), (11), and (14) are not true. Only the strict preference relation would be transitive, i.e. formula (4) is true.

In the case of $c$ alternatives, finding the ordering under non-transitivity is not an easy task. Let the alternatives be sorted in descending order of their medians. Let $\operatorname{ord}(z)$ be the number of the alternative at the $z$-th place in this ordering:

$$
\text { med }_{\text {ord }(1)} \geq \operatorname{med}_{\text {ord }(1)} \geq \ldots \geq \operatorname{med}_{\text {ord }(c)}
$$

Then, if $p_{\text {value }}(\operatorname{ord}(k+1), \operatorname{ord}(k))<\alpha \quad$, then $b_{\text {ord }(k)} \succ b_{\text {ord }(k+1)}$. Otherwise, $b_{\text {ord }(k)} \sim b_{\text {ord }(k+1)}$. This rule is applied for $k=1,2, \ldots, c-1$. In the resulting multiple preference order, among all alternatives between each two consecutive alternatives there is indifference or strict preference. Let's denote the count of the strict preference signs as $R$. For example, for $c=10$ one possible result is:

$$
\begin{aligned}
& b_{\operatorname{ord}(1)} \succ b_{\operatorname{ord}(2)} \sim b_{\operatorname{ord}(3)} \sim b_{\operatorname{ord}(4)} \succ b_{\operatorname{ord}(5)} \sim \\
& \sim b_{\operatorname{ord}(6)} \succ b_{\operatorname{ord}(7)} \sim b_{\operatorname{ord}(8)} \sim b_{\operatorname{ord}(9)} \sim b_{\operatorname{ord}(10)}
\end{aligned}
$$

Then $R=3$. From each multiple preference order, $R+1$ pseudo-indifference classes $I^{p}$ can be formed as follows: the first pseudo indifference class $I_{1}^{p}$ starts at the beginning of the multiple preference order and ends at the first strict preference sign. The $i$-th pseudo indifference class $I_{i}^{p}$ for $\mathrm{i}=2,3, \ldots, r$, would contain the alternatives between the $i-1$-th and the $i$-th strict preference signs. The last pseudo-indifference class $I_{R+1}^{p}$ would contain the alternatives after the last strict preference sign. Of course, if in the multiple preference order, there are only indifference signs, then all alternatives belong to one pseudo indifference class by the alternatives that lay between each two consecutive strict preferences. For the above example, there are four indifference class $I_{1}^{p}$.

For the example given above, $I_{1}^{p} \succ I_{2}^{p} \succ I_{3}^{p} \succ I_{4}^{p}$, where $\quad I_{1}^{p}=\left\{b_{\operatorname{ord}(1)}\right\} \quad, \quad I_{2}^{p}=\left\{b_{\operatorname{ord}(2)}, b_{\operatorname{ord}(3)}, b_{\operatorname{ord}(4)}\right\}$, $I_{3}^{p}=\left\{b_{\text {ord }(5)}, b_{\text {ord }(6)}\right\}, I_{4}^{p}=\left\{b_{\text {ord }(7)}, b_{\text {ord }(8)}, b_{\text {ord }(9)}, b_{\text {ord }(10)}\right\}$.

Between each two elements from two different indifference classes, there is strict preference, exactly as the case is with the indifference classes. However, it is not possible to say what the relation between each two alternatives in a single pseudo-indifference class is - strict preference or indifference, unlike the indifference classes where there is indifference between all objects in the class. However, each alternative of a given pseudo-indifference class is indifferent at least to one of the other alternatives in this class, exactly as with indifference classes. In a sense, the pseudo indifference classes are a generalization of classical indifference classes. An interesting property of pseudo indifference classes is that when they contain one or two elements, they actually transform into indifference classes. Another interesting property is that when they contain at least three elements, two of them at most may have less than two elements from the pseudo indifference class to which they are indifferent.

\section{Numerical experiment}

The REPOMP procedure (Randomized Expert Panel Opinion Marginalizing Procedure) analyzes the subjective opinion of an expert panel in multi-criteria decision making situations. In the example, the REPOMP procedure is employed to analyze the progress of work in the national spatial data infrastructures of 26 countries from the EU. Initial screening has outlined only 13 out of these 26 countries to be subjected to analysis. The methodology is based on expert estimates regarding the significance of a hierarchy of criteria, and the assessment of each country against those criteria. As a result of the analysis, two countries should be outlined and visited in order to exchange good practices in the elaboration of the 
spatial data infrastructure. A detailed description of this example is provided in [Ivanova et al., 2013]. A grouping in pseudo indifference classes shall be conducted with the help of the approaches discussed in the previous sections.

The REPOMP procedure has five steps of application, which follow consecutively below.

1. Expert definition of a hierarchy structure of primitive (directly estimated) and marginal (calculated on the basis of their components' estimates) criteria;

The hierarchy of criteria defined with the help of six experts divides the main marginal criterion $K$ into $b=3$ base marginal criteria: $K_{1}$ - "Quality of the country's system", $K_{2}$-"Benefits from the visit", $K_{3}$ - "Technical aspects of the visit" and each of them is divided into more marginal criteria.

2. Estimating the significance coefficients for each criterion by each expert.

For each of the criteria the experts have given estimates for their coefficient of significance according to the following scale: 0 - criterion with no significance for the case; 1 - criterion with very low significance for the case; 2 - criterion with low significance for the case; 3 criterion with medium significance for the case; 4 criterion with high significance for the case; 5 - criterion with extremely high significance for the case.

3. Estimating alternatives against the primitive criteria by each expert

The experts have evaluated all the countries according to the degree to which they fulfill the requirements stated in the directly estimated criteria. The ranking scale, which is used, is from 1 to 9, the latter being the highest estimate. Considering that, the preferred alternative is the one with the highest total marginal indicator.

4. Calculating the total marginal criterion (ranking ball) for each alternative

In this stage, the estimates of the experts from steps 2 and 3 are combined in order to calculate the total marginal indicator for each alternative. At each hierarchy level the coefficients of significance are used as weight coefficients to the experts' estimates on how the alternatives meet the criteria.

5. Calculating the ranking ball standard deviation for each technology

Since the actual panel consists of a random sample from the general population of all possible experts, then the acquired ranking ball value is an actual estimate. In another similar experiment, another ranking ball shall be obtained. In a hypothetic world, a great number $(M)$ of similar experiments can be performed in order to obtain a great number of point-estimates of the ranking ball, and eventually find the characteristics of its distribution. REPOMP applies the Bootstrap modification of Monte Carlo, described in [Efron, Tibshirani, 1993]. The essence of the Bootstrap method is in the generation of a great number $M$ of synthetic learning samples with a structure identical to the one of the actual learning sample, which are obtained from the latter by drawing with replacement. As a result, $M$ number of synthetic ranking balls are obtained. A pseudo-reality is built, where the actual estimates of the true parameters replace the true parameter.

Figure 1 shows the densities of alternative's ball estimates and Table 1 shows a summary of the results of all the countries obtained with $M=5000$ Bootstrap experiments, including ranking ball, mean value and standard deviation.

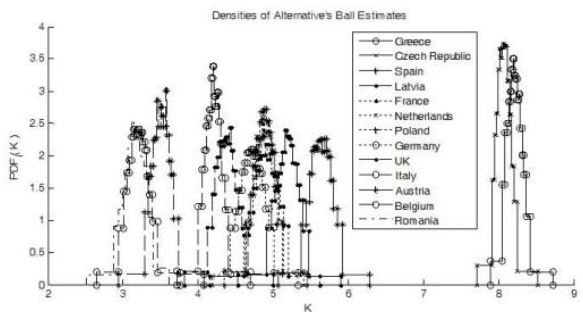

Fig. 1. Densities of the alternative's ranking ball estimates

Table 1. Summary of the results, obtained with $M=5000$ Bootstrap experiments

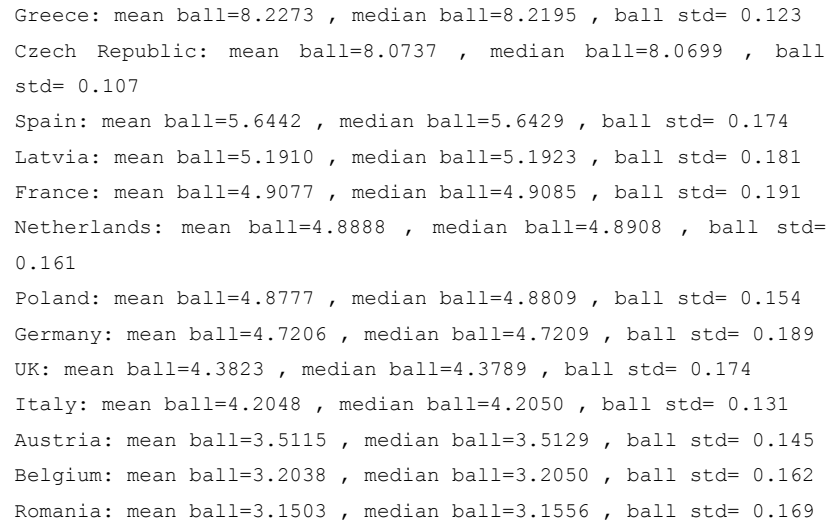

The significance of the difference between each two consecutive alternatives from the ranking may be analyzed by testing the null hypothesis $H_{0}$ - "alternative $i$ is indiscernible from alternative $j$ " and the alternative hypothesis $H_{1}$ - "alternative $i$ is discernible from alternative j". There is a probability of making an error while separating two consecutive alternatives from the ranking. If the critical probability for the separation error is $p_{\text {crit }}$, then for each two alternatives for which the $p_{\text {value }}$ does not exceed $p_{\text {crit }}$, the null hypothesis is rejected and the alternative one is accepted, i.e. they are discernible (alternative $i$ is better than $j$ ). When the $p_{\text {value }}$ exceeds $p_{\text {crit }}$, the null hypothesis is accepted, i.e. the alternatives are indiscernible. Having that in mind, Fig. 2 shows the alternatives, grouped in pseudo indifference classes at $p_{\text {crit }}=0.05$, based on the information from Table 2 . 
Table 2. Resulting preferences from the hypothesis tests at $p_{\text {crit }}=0.05$

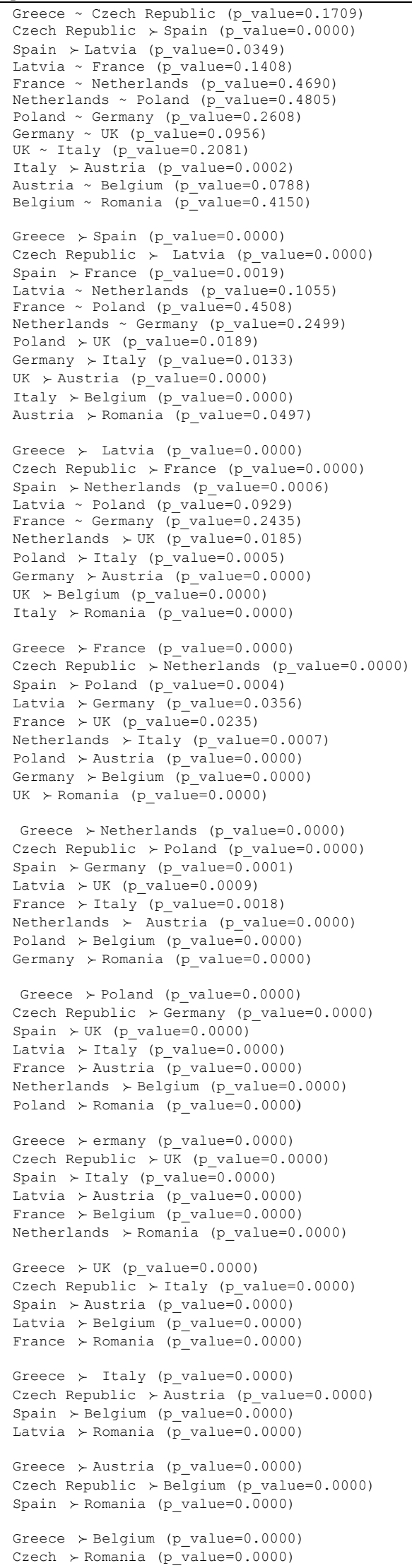

Greece $\succ$ Romania (p value $=0.0000)$

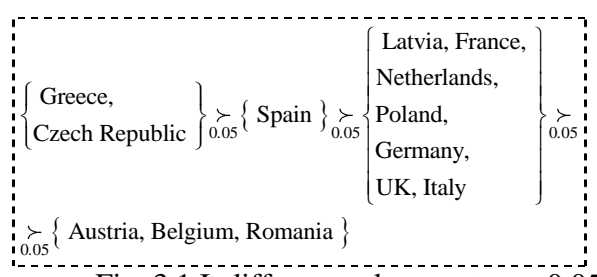

Fig. 3.1 Indifference classes at $p_{\text {crit }}=0.05$

The same analysis is performed at a significance level of $p_{\text {crit }}=0.001$, in order to compare how the groups of alternatives vary with the change of $p_{\text {crit }}$. The results are given in Table 3 and on Fig. 3.1 and 3.2

Table 3. Resulting preferences from the hypothesis tests at $p_{\text {crit }}=0.001$

Greece Czech Republic (p_value=0.1709)

Czech Republic $\succ$ Spain (p value $=0.0000$ )

Spain Latvia (p value $=0.0349)$

Latvia France ( $\bar{p}$ value $=0.1408$ )

France Netherlands ( $p$ value $=0.4690)$

Netherlands Poland ( $\mathrm{p}$ value $=0.4805$ )

Poland Germany (p value $=0.2608$ )

Germany UK (p_value $=0.0956$ )

UK Italy (p value=0.2081)

Italy $\sim$ Austría (p_value=0.0002)

Austria Belgium (p_value $=0.0788$

Belgium Romania (p_value $=0.4150$ )

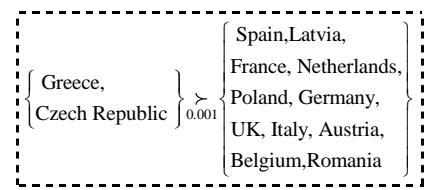

Fig. 3.2 Indifference classes at $p_{\text {crit }}=0.001$

At a significance level of $p_{c r i t}=0.15$, the resulting rankings are given by Table 4 and on Fig. 4 .

Table 4. Resulting preferences from the hypothesis tests at $p_{\text {crit }}=0.15$

Greece Czech Republic (p_value $=0.1709$ )

Czech Republic $\succ$ Spain (p_value=0.0000)

Spain $\succ$ Latvia (p value $=0.0349$ )

Latvia $\succ$ France (p value $=0.1408$ )

France Netherlands ( $p$ value $=0.4690$ )

Netherlands Poland (p value $=0.4805$ )

Poland $\sim$ Germany (p value $=0.2608)$

Germany $\succ$ UK (p_value $=0.0956)$

UK Italy (p value $=0.2081)$

Italy $\succ$ Austria (p value $=0.0002$ )

Austria $\succ$ Belgium ( $\mathrm{p}$ value $=0.0788$ )

Belgium Romania (p value=0.4150)

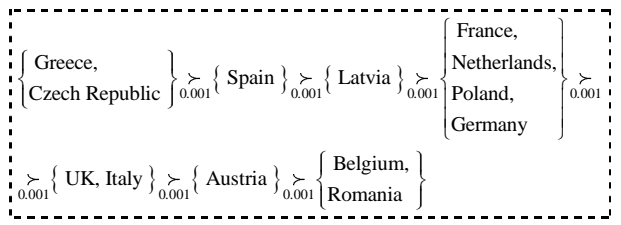

Fig. 4. Indifference classes at $p_{c r i t}=0.15$

At a significance level of $p_{\text {crit }}=0.30$, the resulting rankings are given by Table 5 and on Fig. 5 . 


\section{Conclusions}

This paper had its main contribution in presenting an approach to rank multiple alternatives with the use of pseudo indifference classes. Those resulted from the random character of the quantities of the value function for the alternatives in some cases, e.g. in computer intensive analysis. In order to analyze the way to put the alternatives into groups, hypothesis tests were proposed in the paper. Following the results from the tests, it was shown that since the resulting binary relation of strict preference finally turned out to be non-transitive, the final content of the pseudo indifference classes strongly depended on the significance level of the comparison. This statement is clearly shown in the example from Section 5 , where the values of $0.05,0.001,0.15$, and 0.30 were selected as significance values. The first two best options (Greece and Czech Republic) wall into different pseudo indifference classes only when the significance level has higher values (e.g., $p_{\text {crit }}=0.30$ ).

Table 5. Resulting preferences from the hypothesis tests at $p_{\text {crit }}=0.30$

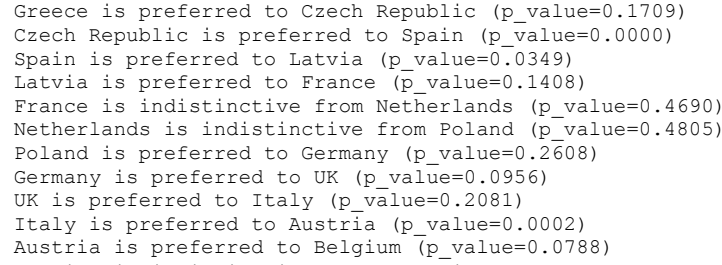

Fig. 5. Indifference classes at $p_{\text {crit }}=0.30$

\section{Acknowledgments}

The preparation of this paper is financially supported by the ERASMUS MUNDUS project 545867-EM-1-2013-1DE-ERA MUNDUS-EMA21 - EUREKA SD, entitled "Enhancement of University Research and Education in Knowledge Areas useful".

\section{References}

[1] Daniels, J. Micro Models. University of Delaware, College of Business and Economics, 1999 http://medusa.be.udel.edu/MicroModels/Indifference.htm

[2] Efron, B., R. J. Tibshirani. An Introduction to the Bootstrap. Chapman \&Hall, 1993.

[3] Ekenberg, L. Order and Value Assignment. Mid Sweden University, Sweden, Hosten, 1998 http://www.ite.mh.se/ love/ba2/preforder2.html [4] French, S. Decision Theory: an Introduction to the Mathematics of Rationality, 1993 Ellis Horwood.

[5] Ghirardato, P., M. Marinacci. The impossibility of Compromise: Some Uniqueness Properties of Expected Utility Preferences. California Institute of Technology, Universita di Bologna, 2000 http://masada.hss.caltech.edu/ paolo/uniqwp.pdf [6] Gilboa, I., D. Schmeidler, P. P. Wakker. Utility in Case-Based Decision Theory, Journal of Economic Theory, Vol. 105, pp. 483-502, 2002.

[7] Ivanova, S., Nikolova, N.D., Kalinov, K., Mednikarov, B., Tenekedjiev, K., Selection of Adequate Models for Development of Spatial Data Infrastructure using REPOMP, Proc. First International Conference of Black Sea Association of Maritime Institutions (BSAMI) "BSAMI 2013", 25-27.06.2013, Admiral Ushakov State Maritime University, Novorossiysk, Russia, 2013 (in print) [8] Sugden, R. The Consistency or Inconsistency of Preferences under Risk and Over Time: Summary of Findings. An Economic and Social Research Council Research Programme (ESRC), School of Economics and Social Studies, University of East Anglia, 1999.

http://www.ukc.ac.uk/ESRC/sugrep.htm.durgo

[9] Sung, Y. Individual Choice and Preferences. National Taiwan University, 2000. http://www.cc.ntu.edu.tw/ ysung/articles/PoliEcon/ch1.pdf.

[10] Tenekedjiev, K., S. Kamenova, N.D. Nikolova. Formalized Procedure for Ranking Alternatives in Developing Environmental Programs, Journal of Cleaner Production, Elsevier, 12(4), 353-360, 2004. 